\title{
Process Modeling for Internet Scale Virtual Enterprise Collaborations
}

\author{
Chong Wang ${ }^{1}$, Lai Xu ${ }^{2}$, Paul de Vrieze ${ }^{2}$, and Peng Liang ${ }^{1}$ \\ ${ }^{1}$ State Key Lab. of Software Engineering, Wuhan University, China \\ \{cwang, liangp\} @ sklse.org \\ ${ }^{2}$ Software Systems Research Centre, Bournemouth University, UK \\ $\{1 \mathrm{xu}$, pdvrieze\} @bournemouth.ac.uk
}

\begin{abstract}
The ever-increasing need for flexibility of business collaborations that ultimately involve a large number of virtual enterprises puts demands on their design. Competitive markets require the collaborations to be highly agile, effective, and efficient. This paper defines Internet scale virtual enterprise collaborations (ISVECs) as well as their characteristics. We further present a process modeling method for Internet-based virtual enterprise collaborations. An end user process modeling language of ISVECs and a meta-model of the language are provided. Finally, we demonstrate how the method and language are used.
\end{abstract}

Keywords: Internet scale business process, Business process modeling, Virtual enterprise process collaboration, Web-scale workflow.

\section{Introduction}

In recent years, the service-oriented architecture paradigm has provided an easy approach to software development. Virtual enterprise systems are increasingly depending on and functioning as driver for the development of large scale distributed applications by reusing services [5].

Internet scale virtual enterprise collaborations (ISVECs) are business collaborations among virtual enterprises. This normally involves great numbers of services available through the Internet. These services should be annotated with semantic descriptions enabling dynamic selection, resource binding, and verification. As the services are provided by different providers, there is a strong likelihood that some of these services are modified, substituted, or disappear. Different versions of the services may include different features or have incompatible semantics. The services can be invoked from outside their own infrastructure, i.e. from a business partner's site. The semantic annotations can help detecting these changes and provide an early warning when the changes are incompatible.

Internet scale virtual enterprise collaborations are possible due to service that exist both inside and outside a virtual enterprise. Any ISVEC architecture should be a service-based development approach with a range of services that represent capabilities across a wide variety of domains. 
As a business environment changes rapidly, the ability to set up a collaborative business process in a virtual enterprise is desirable [8]. Collaborative business processes are increasingly driven by business agility, adaptability, and flexibility, particularly in a virtual enterprise environment. There is increased pressure to build enterprise applications quickly in order to respond to situational needs of the business.

In this paper, we deal with lightweight business process modeling issues. We start with a motivating example. We define a lightweight business process modeling language and provide its meta-model. The paper also uses the lightweight business process modeling language to model a motivating example. Finally, conclusions and future research are presented.

\section{Motivating Example: Internet Moving Services (IMS)}

As a motivating example we introduce a hypothetical international moving service. An international moving service aims to facilitate international relocations in various ways. These services go beyond moving items and can include things such as visa applications and assistance in finding a new residence. In brief, the goal of international moving services starts with helping customers to find moving companies and request quotes, but extends to many aspect of managing an international relocation. A very brief, incomplete and abstract description of the various services offered by an international moving service includes:

- Find moving companies: compare the services of international movers by requesting free quotes for the customer; provide moving tips, and information documents needed for international moving such as official government customs, visa and immigration, health, weather, etc.

- Travel arrangements: find cheapest tickets and/or car renting in both places of departure and destination if needed.

- Temporary stay arrangement: find hotels or holiday/serviced apartments in both the departure location and the destination if needed.

- Home search: pre-select properties according to client requirements such as proximity to a childcare centre and provide neighborhood guide which contains information on doctors, shopping, schools, leisure activities etc.

- School/childcare search: provide explanation of the local education system, options including public, private and international schools, provide information on preschool options including nurseries, toddler groups and other childcare facilities, provide list of possible schools, childcare or other facilities relating to the home search area.

- Settling-in services: advice on banking systems, provide information on insurance of health, home, car etc., and advise on importing a car into the destination if applicable.

- Leaving assistance: arrange property hand-back or sale, close utility accounts and arrange final bills, and manage property if client leaves before end of tenancy.

We have found the following available Web services, feeds, widgets, and mashups from websites like syndic8.com and programmableweb.com. A list of available feeds, 
Web services, widgets, gadgets, mashups that can be used as components in implementing the example:

1. Moving company feeds from 123 movers.

2. Moving tip feeds from 123 movers.

Both Feeds 1 and 2 can support the service of "find moving companies"

3. SmartTravelDeals: publishes the best travel deals on the web. It publishes current travel offers to worldwide destinations and connects users to the direct booking path.

4. Hotwire Travel-Ticker Deals: connect with more than 10,000 travel companies. The deals can be sceached by destination, theme, dates, or price.

Both API 3 and 4 sustain the service of "travel arrangements"

5. Cleartrip Hotel API: provides booking for flights, hotels and trains, as well as other travel services across the world.

6. Active.com Camping API: provides access to campground data for $97 \%$ of the US and Canada's national and state/provincial parks.

7. HomeFinder.com connects home buyers, sellers and real estate professionals through local newspapers' online real estate section.

8. ImmobilienScout24 API provides rental listings, house listings, and building construction information from Germany.

9. PeekaCity API: using google street view for neighborhood amenities which is used primarily by real estate agents as a service to their customers (currently in Chicago and Dallas/Fort Worth).

10. Easy one loan and home values: mashup of zillow and yahoo maps as supplement to on-line mortgage service.

Above five APIs can be included to implement the service of "temporary stay arrangement"; API 5,7,8, 9 and 10 can be used for the service of "home search".

11. Child care finder mashup: find babysitters, nannies, and other care options visually with google maps

12. California school finder mashup provides information such as, the school rankings, course offerings, and other details.

13. Feed Childcare position offered in France.

14. Feed Childcare position wanted in France

Above API 9, Mashup 11 and 12, and Feed 13 and 14 can be applied to implement the services of "school/childcare search".

15. NHS Choices has created a set of web services to allow approved partners to interact with the service, free of charge.

16. Patient Opinion is a UK organization that collects patients' opinions about heath care and treatments they have recently received.

17. Hospital Compare API allows developers to get various hospital information like addresses, mortality rates, prices for operations and other hospital-related data.

18. Monster Web Services Toolkit API allows users to publish job ads to the Monster.com job search and placement service. Monster service includes job seeking, career management, recruitment and talent management products and services.

Above APIs and Mashups are useful for the "settling in service". 
19. Home value calculator: uses zillow data to calculate the value of single family homes in the U.S. Small widget suitable for placing on your google home page. It is a useful mashup for the service of "leaving assistance"

In this example case, it will be expensive and difficult to build a traditional workflow system to support the business process. Doing so would mean that either all information would have to be known in advance or an interface would need to be provided to add information to the traditional workflow solution. Few relocations would be exactly alike even though aspects could be shared. The dependences are various, such as finding a home close to the best school or the available childcare, find the good schools close to the home address. It would however be handy for a business process mashup solution, specially, if automatically invocation of needed feeds, Web services, etc. and execution processes are supported. The different processes of IMS can be implemented by different process instances. Users (i.e. owner of IMS) may only need to edit the certain processes to be able to meet all requirements from new customers.

\section{Meta-Model of the ISVECs Language}

In this section, we introduce the meta-model of the ISVEC language which the end users can use it to establish the business processes mentioned in Section 2. The notations of the ISVEC language are presented in Figure 1. Eight symbols are adopted from BPMN [1]. The choice of BPMN as basis is motivated by its wide acceptance within the business world. Other alternatives may offer different advantages for different user groups. The ISVEC language is based on workflow patterns [8]. Adoption of different notations should not change the semantic of the languages.

Since the process model is finally mapped into concrete services, we introduce new notations in the form of goals, which will allow business users to define abstract and user-friendly processes. Goals are the representation of an objective in which fulfillment is sought through the execution of a possibly complex service. The goals are always associated with an activity or process. We define two types of goals: atomic goals and composite goals. Atomic goals are associated with a single concrete Web service or computing resource, involving just one step of computation. Composite goals involve other simpler subgoals for fulfillment. In practice, if a process or activity is a process associated with a complex goal then the process or activity is fulfilled by achieving other goals, or invoking composite concrete Web services or computing resources. Hence, there are, in total, ten notational symbols within our lightweight process modeling language. This minimal subset of notation means a user will have reduced learning, whilst, we contend, still providing sufficient expressive power.

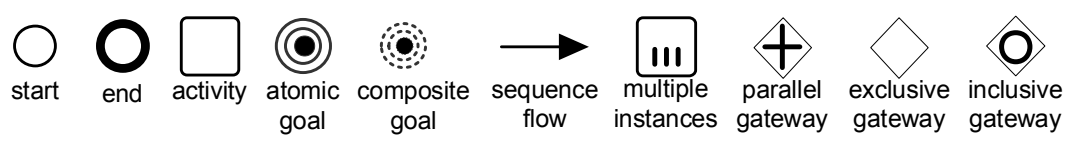

Fig. 1. Notations of Lightweight Process Modeling Language 


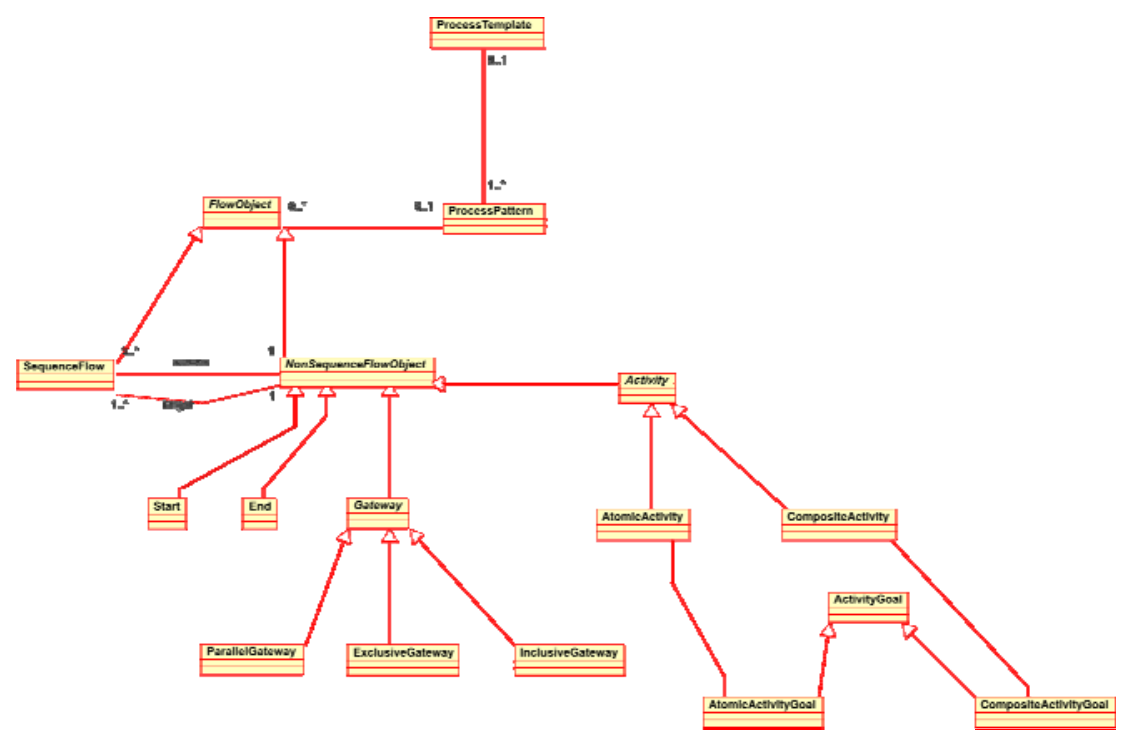

Fig. 2. Meta-model of Lightweight Process Modeling Language

Figure 2 provides a meta-model for the control flow of the lightweight process modeling language. The meta-model describes the relationships among all elements within the process model, namely 'start', 'end', 'activity', 'sequence flow', 'exclusive gateway', 'parallel gateway', 'inclusive gateway', 'atomic goal', and 'composite goal'. An abstract concept 'gateway' is used as a super set of three gateways. An 'atomic activity' is the smallest unit of activity, e.g., a concrete Web service or a computing resource. A 'composite activity' consists of several other activities, either atomic or composite. An 'activity' is either an 'atomic activity' or 'composite activity'. The terms 'process pattern' and 'process template' are explained [8], and 'flow object' in the figure represents a process model. We separate 'non sequence flow object' from 'sequence flow', to ensure the alternating ordering of 'sequence flow,' i.e., arrows and other objects, and thus avoid the situation where two 'sequence flow' elements link directly to each other.

\section{Model of the Motivating Example}

An overview of the services for Virtual Enterprise-Internet Moving Services (VEIMS) can be found from Figure 3. Being a VE-IMS, the payment, CRM, and bookkeeping functions that should be included for being a normal business are sourced from third parties. However, we only concentrate here on the VE's core business processes. General business related processes are not discussed here.

Because of the various requirements from its customers, the services provided by a VE-IMS are dependent on the particular situation of its customers. Different customers require a different process. This process is supported by a special-purpose piece of software, which we call an business process mashup, with particular services, processes or activities. In addition to the added capability, new business process mashup can 


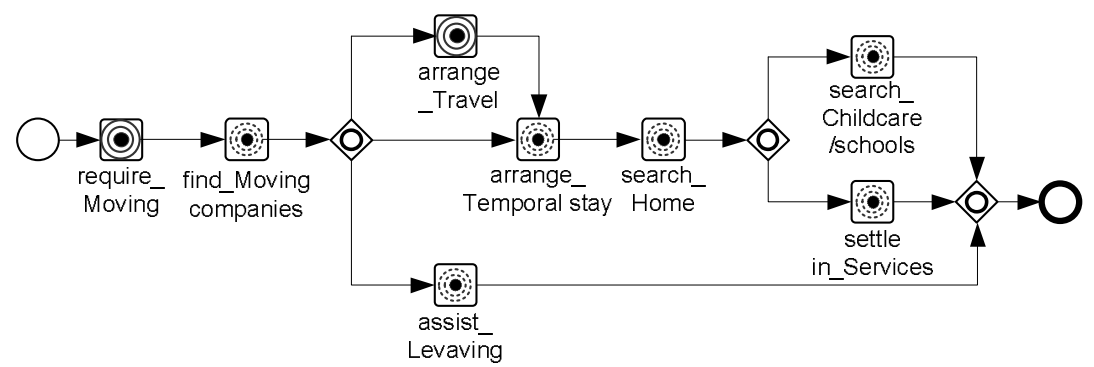

Fig. 3. Meta level services of the VE-IMS

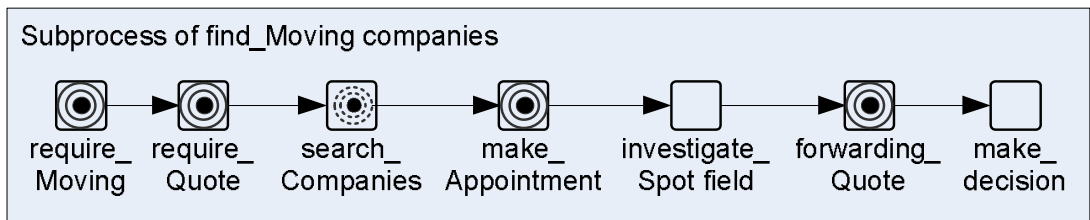

Fig. 4. Subprocess of finding a moving company

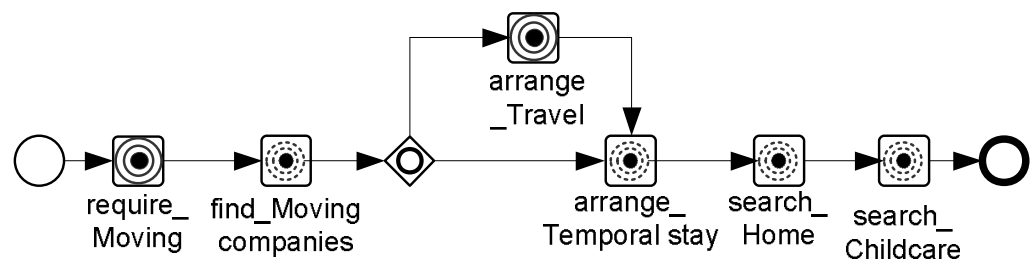

Fig. 5. Processes for the extended VE-IMS

modify, enhance, customize or extend an existing service mashup, or include and combine parts or components (or both) from multiple existing service mashups.

A preliminary service of the VE-IMS is to help customers to find a moving company for shipping their household effects to the new place of residence. Figure 4 shows the process of the finding a moving company. First, the VE-IMS will request free quotes from moving companies according to the customer's place of departure and destination, and if needed, arrange for visits, and provide a list of competent movers with their quotes.

Another, extended, service the VE-IMS may provide is finding an international mover and arranging temporary places of residence at both the locations of departure and destination, based on the dates of moving, travel, and the arrival of the household effects. The temporary place of residence at the destination should be close to a certain address such as the customer's working place. Customer can also ask for travel arrangements to be made. The time to fly and time of staying at the temporary address should be worked out to minimize the total costs. Further, the customer may want the VE-IMS to find an available childcare place for the children of the customer as soon as possible, then find a rental home close by around the time that the household effects arrive. The second extended international moving service is shown in Figure 5. 


\section{Related Work}

The concept of Internet scale workflows is sometimes used interchangeably with that of Web-scale workflows. Both Internet and Web scale workflows involve great number of internet based services and adopt a service-oriented paradigm [2, 4]. However, not all service-based workflows are Internet scale workflows or Web scale workflows. Internet or Web scale workflows involve services distributed though the Internet. There are many open issues related to Internet or Web scale workflows. Lightweight and user friendly process modeling is one of information issues at a design stage. Process modeling further effect to service description and process execution.

Lightweight process modeling language is designed for process oriented business [3]. The language is designed for business users who do not have deep business process design knowledge. It requires the process mashup engineer who could map the abstract activities into the concrete services. Our modeling language for ISVECs is based on the lightweight business process modeling language [3] and lightweight process modeling for virtual enterprise process collaboration language [8]. It provides strong end user aspects on business flexibility, adapbility and agility. The Internetscale virtual enterprise collaboration is therefore different with an approach mentioned in Internet-scale workflow [2]. The process mashup engineer will discover services and map abstract process activities into concrete services [7].

Similarly, the concept of instant virtual enterprises (IVEs) is introduced in [6] to dynamically select collaborating partners and weave the interorganisational links between their local processes. Moreover, CrossWork system is developed to create and operate IVEs by decomposing high-level goal into a set of operational business goals, identifying collaboration partners that can fulfill the goals, retrieving the external specifications of selected local process, and finally mapping the composed global business process onto the IVE's distributed infrastructure and executes it there. Different from creating IVEs, our approach concentrates on the business collaborations among virtual enterprises. Particularly, it proposes a lightweight process modeling language and the corresponding metamodel to create the Internet scale virtual enterprises collaborations, and introduces goals to help business users define abstract processes and finally map them into concrete services available on the Internet.

\section{Conclusions}

Internet scale workflows put more demands on process modeling. Multiple service providers are generally involved, and their actions are largely independent and outside control of the process owner, services can suddenly change because of new versions. Networking issues can make services temporarily unavailable or have unacceptable latencies.

Virtual enterprises have a strong advantage in business agility. Their small size and loose ties with partners make that they are not held back by large bureaucracies. They depend on tight integration with suppliers of various services. Internet and automation enable this integration. Automation of business processes is generally provided by 
business process management systems. For virtual enterprises to be able to quickly respond to changing business environments they therefore need agile business process support.

Agile business process support has two parts. The first part is run-time support for change on-the-fly, a topic not discussed in this paper. The second part is to make it easy to change business processes. To enable business process agility modification of processes should be possible by a broad set of stakeholders, not only by business process specialists as an extra link in the chain.

Business process mashups [7] are designed to provide agility, resilience and end user modifiability. Therefore, they are a good candidate to support internet scale virtual enterprise collaborations.

Acknowledgement. This research project is supported by the National Basic Research Program of China (973) under Grant 2007CB310801, the National Natural Science Foundation of China under Grant No. 60970017, and the Fundamental Research Funds for the Central Universities under Grant No. 3101032.

The authors would like to thank the EU SOA4All and FAST projects. Part of work by Dr. Lai Xu and Dr. Paul de Vrieze was performed at the SAP Research, Switzerland.

\section{References}

1. BPMN, http: / / www . bpmn . org/

2. Tretola, G., Zimeo, E.: Autonomic internet-scale workflows. In: Proceedings of the 3rd International Workshop on Monitoring, Adaptation and Beyond (MONA 2010), pp. 48-56. ACM, New York (2010)

3. Xie, L., Xu, L., de Vrieze, P.: Lightweight Business Process Modelling. In: 2010 International Conference on E-Business and E-Government (ICEE 2010), Guangzhou, China, May 7-9 (2010)

4. Blake, M.B., Huhns, M.N.: Web-Scale Workflow: Integrating Distributed Services. IEEE Internet Computing 12(1), 55-59 (2008)

5. Papazoglou, M.P., Georgakopoulos, D.: Service-oriented Computing. Communications of the ACM 46(10), 24-28 (2003)

6. Grefen, P., Eshuis, R., Mehandjiev, N., Kouvas, G., Weichhart, G.: IEEE Internet Computing 13(6), 65-73 (2009)

7. de Vrieze, P.T., Xu, L., Bouguettaya, A., Yang, J., Chen, J.: Building enterprise mashups. Future Generation Computer Systems 27(5), 637-642 (2011)

8. Xu, L., de Vrieze, P.T., Phalp, K.T., Jeary, S., Liang, P.: Lightweight Process Modelling for Virtual Enterprise Process Collaboration. In: Camarinha-Matos, L.M., Boucher, X., Afsarmanesh, H. (eds.) PRO-VE 2010. IFIP AICT, vol. 336, pp. 501-508. Springer, Heidelberg (2010) 